\title{
Wavelength-Selective Near Unity Absorber Based on Fabry-Pérot Nanoresonators
}

\author{
I. L. Gomes de Souza ${ }^{1}$ (i), V. F. Rodriguez-Esquerre ${ }^{2}$ (i) \\ ${ }^{I}$ Institute of Science, Technology and Innovation, Federal University of Bahia, Camaçari, Bahia, Brazil, \\ ilgsouza@ufba.br \\ ${ }^{2}$ Department of Electrical Engineering, Federal University of Bahia, Salvador, Bahia, Brazil, \\ vitally.esquerre@ufba.br
}

\begin{abstract}
We proposed and analyzed a planar narrow-band absorber based on a bi-layer metal/dielectric structure. The planar absorber uses the Fabry-Pérot resonance and the inherent loss of the metals, for maximum light absorption in the range of the electromagnetic spectrum visible to the near infrared. The absorption resonance peak can be shifted to other regions of the spectrum by varying the dielectric film thicknesses. It is also possible to control the absorption with the angle of incidence for TE and TM polarization modes. The high absorption (near unity) and the high tolerance to fabrication errors of the proposed absorber can be explored in a large number of optoelectronic devices.
\end{abstract}

Index Terms - Electromagnetic wave absorption, Optical resonators, Resonators filters, Optical Filters.

\section{INTRODUCTION}

Absorbers based on metamaterials (MMs) have attracted lot of interest in the last decades due to the vast amount of applications in photodetection, image generation, thermal emission, sensors and shielding [1]. There are two types of MMs absorbers, the broadband that has high/maximum absorption for all wavelengths of a region of the electromagnetic spectrum and the narrow-band that has maximum absorption for a given wavelength [2]. Recently, ultranarrowband perfect absorbers have been studied [3]-[5]. Resonant structures composed by metals and dielectrics with subwavelength dimensions have been used as mirrors to suppress transmission by increasing the reflection and inducing destructive interference from reflected light at resonant wavelengths that are absorbed within the structure [6]. With the possibility of joining the knowledge of plasmonics with MMs, it was possible to design, fabricate and characterize ultra-fine, highly efficient absorbers that can be coupled to optical-electronic nanoscale circuits [7]. Perfect absorbers (PAs) are structures that can achieve complete annihilation of the incident electromagnetic waves by eliminating all reflection and transmission [8]. PAs based devices are widely studied in engineering as they have great potential for practical applications and have attracted a lot of attention due to their excellent ability to harvest electromagnetic energy. In [9], a PA for multiple band light absorption in the region of the visible and ultraviolet was proposed and demonstrated, the absorption is obtained by optical resonances supported by the high index dielectric cavity with values of $98.9 \%$ for multiple resonance peaks. A semiconductor meta-surface light absorber, consisting of a matrix of silicon patches in a thin silicon 
film and an opaque silver substrate, was proposed in [10]. Using the effect of Mie resonances and the fundamental cavity mode of the silicon film, it was possible to obtain a perfect multiband absorption with a peak value of $99.5 \%$, the absorption behavior of the structure can be scalable by adjusting the structural parameters. The main characteristics to design a PA with almost $100 \%$ absorption has direct correspondence with the choice of the materials where the resulting impedance of the structure is approximately equal to the one of free space. Consequently, the reflection will be null resulting in maximum absorption [11]. The design of perfect absorbers based on MMs that operate in the infrared visible region, usually requires complex geometries, making the fabrication process complex because of the sub-wavelength dimensions of the structures.

One possible solution to this problem would be a simple fabrication process using planar deposition of thin metal/dielectric films, this type of design does not require expensive neither complex fabrication techniques. Fabry-Pérot (FP) planar resonator [12], is a tri-layer Metal/Dielectric/Metal (MDM) structure, the choice of the metal and the dielectric as well as its thickness produce resonances that can be applied in several electronic optical devices such as light transmitions filters [13], photodetectors, optics amplifiers, photovoltaic cells phototransistors [5] and antennas [12].

In this work, we propose and numerical analyze a narrow-band FP absorber for visible and nearinfrared frequencies, exploring the theory of FP planar resonators [11]. We analyzed the behavior of the structure with the variation of the dielectric thickness, investigated the physical mechanism of coupling of the electromagnetic field in the resonant cavity and the dependence of the resonance with the angle of incidence for transverse electric (TE) and transverse magnetic (TM) polarizations. This kind of resonators are being extensively studied for absorption [6], [7], [15] and transmission [13], [16] of light in the visible spectrum and in Infrared (IR) [4], [5],[12]. The metallic layers in the planar nanoresonators are considered to extend to the semi-infinite.

\section{ABsorber Design ANd Methodology}

We assume that the metal and dielectric of the nanoresonator are non-magnetic $\left(\mu_{1}=\mu_{2}=\mu_{0}\right)$ and that the dielectric used in the design is lossless [16]. Here the Silver (Ag) was chosen as the metal of the nanoresonator, mainly because it has low optical absorbance when compared to other noble metals [2]. Figure 1a shows the planar FP MDM absorber, which is composed of a thin layer of silver (Ag), Silicon Nitride $\left(\mathrm{Si}_{3} \mathrm{~N}_{4}\right)$ and copper $(\mathrm{Cu})$ substrate.

If the upper $\mathrm{Ag}$ layer is too thick the light is reflected [16], the optimum thickness for maximum absorption should be adjusted to allow the coupling of the light at the resonant wavelength into the resonant cavity. For the operating frequencies, Ag penetration depth is in the range of 10-50 nm [17]. The substrate is considered thick enough, consequently, the transmission is zero. The Finite Element Method (FEM) [18] is used to calculate the reflection $(R)$ and transmission $(T)$ coefficients of the structure. 


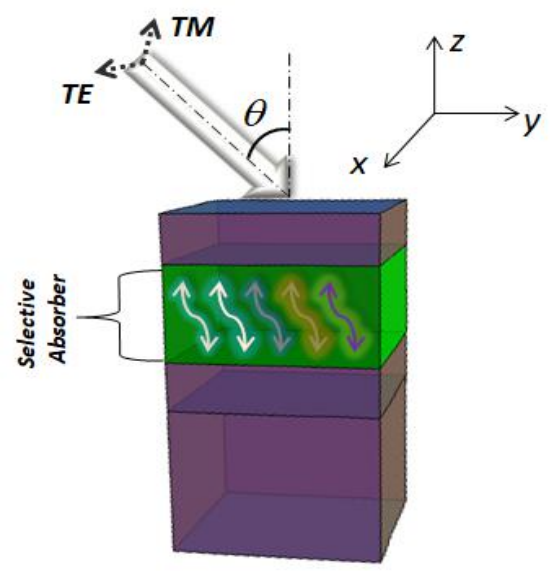

(a)

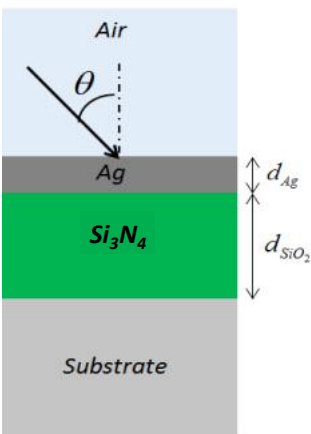

(b)

Fig. 1. Planar FP metal dielectric absorber.

For the calculation of the $\mathrm{Si}_{3} \mathrm{~N}_{4}$ refractive index at each wavelength of the electromagnetic spectrum analyzed, the dispersion relation given by (1) is used [19].

$$
n^{2}-1=\frac{3.0249 \lambda^{2}}{\lambda^{2}-0.1353406^{2}}+\frac{403.14 \lambda^{2}}{\lambda^{2}-1239.842^{2}}
$$

For the real and imaginary permittivity of Ag we use the data given by [20].

Fig. $1 \mathrm{~b}$ shows the cross-section of the absorber in two-dimensional (2D) with the geometric parameters, the $\mathrm{Ag}$ and $\mathrm{Si}_{3} \mathrm{~N}_{4}$ thicknesses of the structure were optimized to have a maximum resonant absorption at $\lambda_{\text {res }}=694.8 \mathrm{~nm}$, the optimum thickness values are $d_{\mathrm{Ag}}=29 \mathrm{~nm}$ and $d_{\mathrm{Si3N} 4}=123 \mathrm{~nm}$.

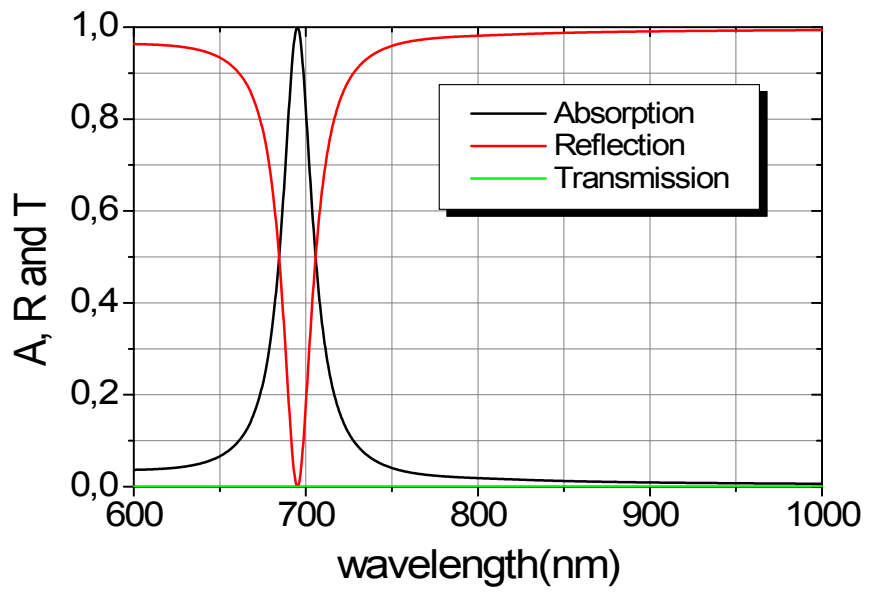

Fig. 2. Absorption (black curve), reflection (red curve) and transmission (green curve) of the structure with values $d_{A g}=29 \mathrm{~nm}$ and $d_{S i 3 N 4}=123 \mathrm{~nm}$.

\section{NUMERICAL RESULTS AND DISCUSSION}

Numerical simulations were performed using a two dimensional frequency domain finite element method [18] to calculate the reflection and absorption and electric and magnetic field distributions of each structure. A plane wave was used as the light source. The absorption of the structure was 
calculated using $A(\lambda)=1-R(\lambda)-T(\lambda)$, the numerical results can be seen in Fig. 2. It is possible to observe an approximate absorption of $97.5 \%$ at the wavelength of $694.8 \mathrm{~nm}$ for a normal incidence $\theta=0^{0}$, the FWHM (full width at half maximum) is approximately $19 \mathrm{~nm}$, showing the high quality of the resonance. The numerical results show that the proposed MDM absorber reflects most of the incident wavelengths, absorbing only a small range of the analyzed spectrum that is almost completely confined in the cavity because of FP resonance. We also analyze the dependence of the absorption with the thickness of the layer of $\mathrm{Si}_{3} \mathrm{~N}_{4}\left(d_{\mathrm{Si} 3 \mathrm{~N} 4}\right)$ the results are presented in Fig. 3. We can observe that the resonances can be tunned by changing the thickness of $d_{\mathrm{Si} 3 \mathrm{~N} 4}$, an interesting characteristic that can be observed is the linear dependence of the resonant frequency with the thickness of the dielectric, using this characteristic of the proposed structure is sintonized to the resonant wavelength with the thickness using (2).

$$
\lambda_{\text {res }}\left(d_{S i_{3} N_{4}}\right)=3.72 d_{S i_{3} N_{4}}+237.41
$$

We can use (2) to estimate the thickness of the dielectric to obtain the resonance at a given spectral wavelength in the approximate range of 500-2000 $\mathrm{nm}$.

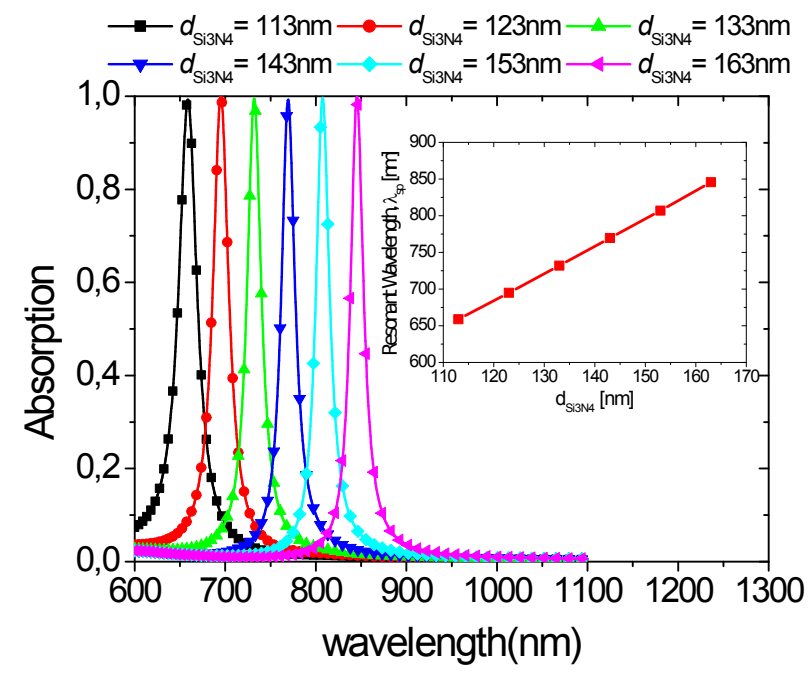

Fig. 3. Absorption as a function of wavelength and the thickness of middle dielectric layer.

In order to better analyze and understand the physical mechanism of absorption in the three-layer MDM cavity, we calculated the spatial distribution of the electric field $(\boldsymbol{E})$ and magnetic field $(\boldsymbol{H})$ (Fig. 4) at the cavity absorption peak resonance shown in Fig. 2. 


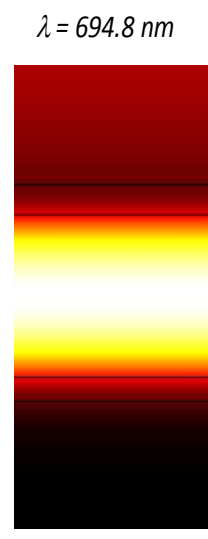

(a)

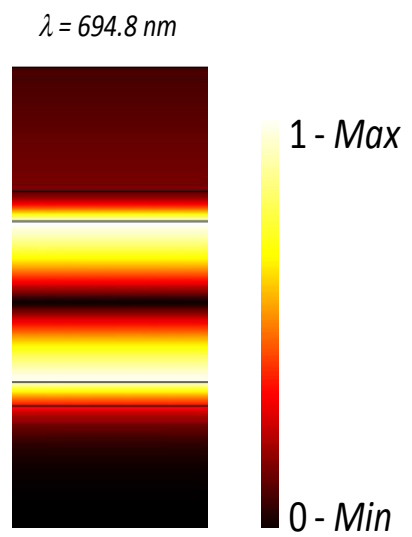

(b)

Fig. 4. Spatial distribution of the (a) electric and (b) magnetic field at the resonant frequency with $d_{A g}=29 \mathrm{~nm}$ and $d_{\text {Si3N4 }}=123 \mathrm{~nm}$.

It is possible to observe that the $\boldsymbol{E}$ field (Fig. 4a) is highly confined in the dielectric between the two metal films, where a standing wave is formed due to the constructive interference [21] of the transmitted and reflected waves. For the $\boldsymbol{H}$ field (Fig. 4b) the absorption map shows that absorption is high at the metal/dielectric interfaces (both at the upper and lower interfaces), this fact is attributed to the excitation of Surface Plasmons Polaritions (SPPs) that occur for transversal magnetic polarization - TM [2], [4] and [21]. The coupling mechanism of the absorber FP is shown in Fig. 5. The condition for FP resonance occurs when (3) is satisfied [14]

$$
\varphi_{1}+\varphi_{2}+k_{2 z} d_{2}=m \pi
$$

here, $m$ is an integer and $k_{2 z}=\omega\left(\varepsilon_{r 2} \mu_{r 2}-\sin ^{2} \theta\right)^{1 / 2} / c$. When (3) is satisfied, the resonance FP occurs, increasing the electromagnetic field within the dielectric layer.

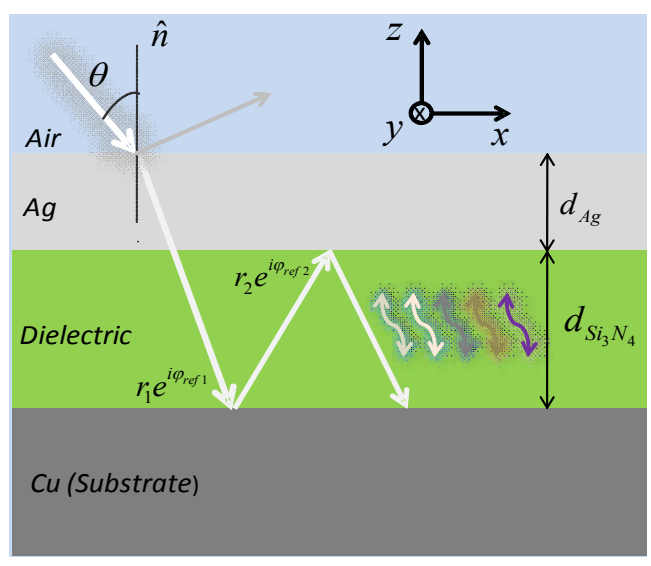

Fig. 5. Physical coupling mechanism of the nanoabsorber FP, incorporating a phase compensation overlap, $\varphi_{1}$ and $\varphi_{2}$ are the reflection phase shift at the two metal-spacer interfaces, respectively.

This fact occurs because the aggregate reflection of the phase changes $\left(\varphi_{1}\right.$ and $\left.\varphi_{2}\right)$ at the upper and substrate metal dielectric interfaces and the phase shift of the wave propagation in the perpendicular direction to the resonator interfaces, results in a phase change of approximately $2 \pi$ in each round-trip 
[23]. In this way, constructive interference and maximum coupling of the electromagnetic field occurs with high absorption and minimum reflection of the light at specific resonant wavelengths [16], [17].

The sensitivity of the proposed nanoresonator has been analyzed by varying the metal thickness in $\pm 2 \mathrm{~nm}$. The results are shown in Fig. 6a-f, for different values of dielectric thickness in the interval [113 nm, $163 \mathrm{~nm}]$ in $10 \mathrm{~nm}$ steps. It can be observed a shifting of about $2 \mathrm{~nm}$ in the resonant frequency and absorption variation of $2 \%$. These features not only show the crucial parameters for the fabrication process of the proposed absorber platform but also indicate a way for a fine tuning of the optical properties.

In order for the resonator to have a high resonance quality, i.e. a low FWHM the ideal choice of metal top thickness is one of the main phases of the design. For a lossless FP resonator, the phase difference at the half maximum is given by (4) [7].

$$
\Delta \delta=2\left(1-R_{0}\right) / R_{0}^{0.5}
$$

Where $R_{0}$ it is the reflectivity of a given metallic mirror. This fact indicates that the FWHM becomes narrower when $R_{0}$ increases. This conclusion is still true for a low loss condition $\left(\Delta \delta^{\prime} \alpha \Delta \delta\right)$, causing a narrower range of absorption when the upper metal thickness is thicker (high reflectivity).

The coupling between the cavity mode and the incident light can be controlled simultaneously by the thickness of the upper Ag layer.

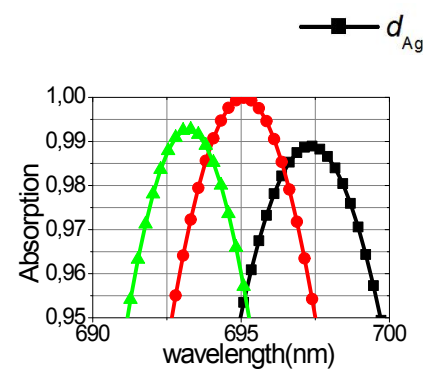

(a)

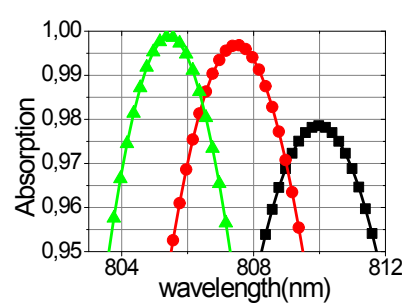

(d)

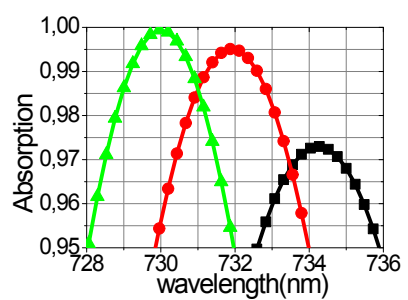

(b)

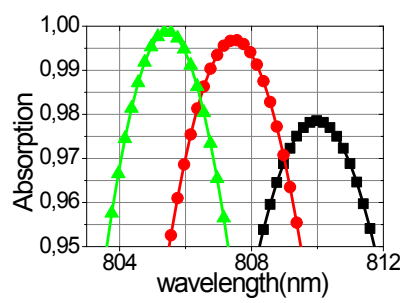

(e)

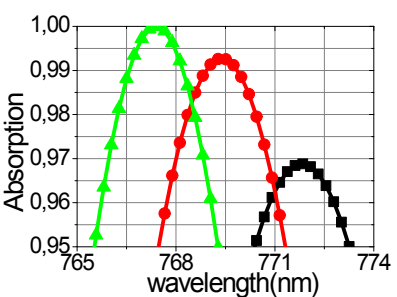

(c)

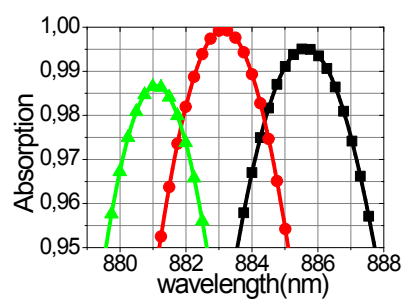

(f)

Fig. 6. Sensitivity of the absorption peak with the top Ag layer thickness $d_{\mathrm{Ag}}$. 
0

$d_{\mathrm{Si} 3 \mathrm{~N} 4}=113 \mathrm{~nm}$

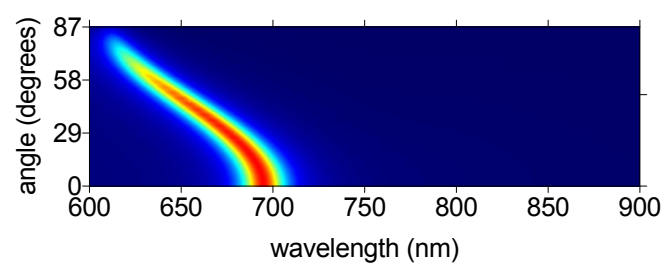

(a)

$d_{\mathrm{Si3N} 4}=133 \mathrm{~nm}$

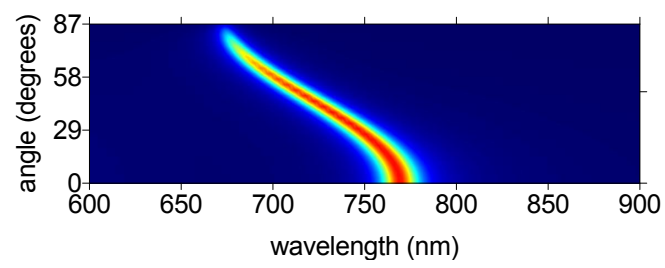

(c)

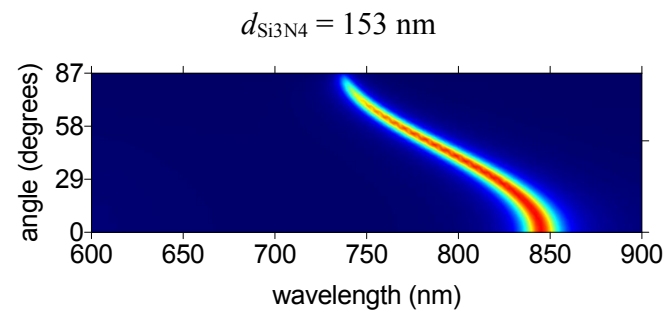

(e)
1

$d_{\mathrm{Si} 3 \mathrm{~N} 4}=123 \mathrm{~nm}$

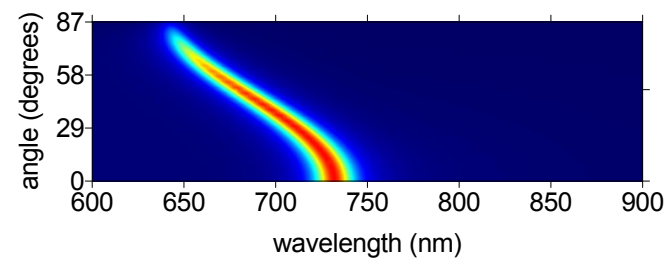

(b)

$d_{\mathrm{Si} 3 \mathrm{~N} 4}=143 \mathrm{~nm}$

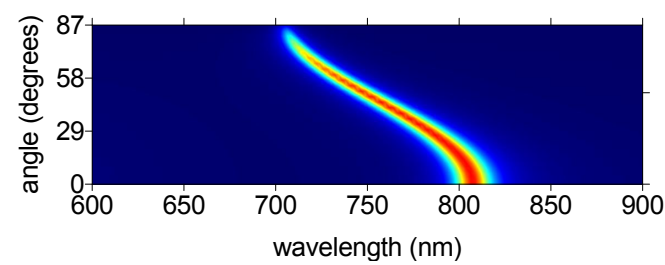

(d)

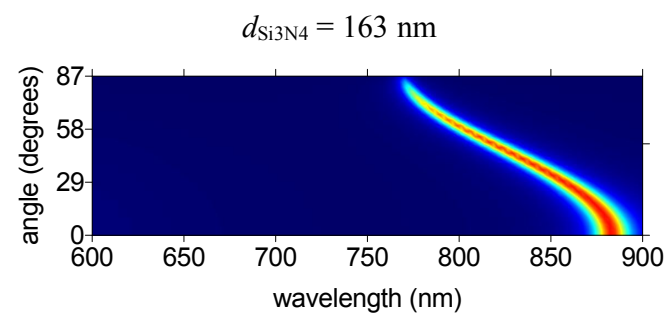

(f)

Fig. 7. Absorption as a function of the incidence angle and wavelength for TE polarization for several dielectric layer thicknesses.

For this type of absorbers, the absorption is highly dependent with the angle of the incidence due to the FP resonance. The absorption as function of the dielectric thickness $\left(d_{S i 3 N 4}\right)$, the incident angles and wavelengths for TE and TM modes are shown in Figs. 7 (a)-(f) and Figs. 8 (a)-(f), respectively, for different values of dielectric thickness in the interval [113 nm, $163 \mathrm{~nm}]$ with $10 \mathrm{~nm}$ steps. Fig. 7 shows that the dielectric thickness has a strong impact on the absorption resonance wavelengths a blue-shift of the absorption peak is observed when the incident angle increases for both modes. For the TM polarized wave, Fig. 8, the resonant wavelength varies more sharply. 


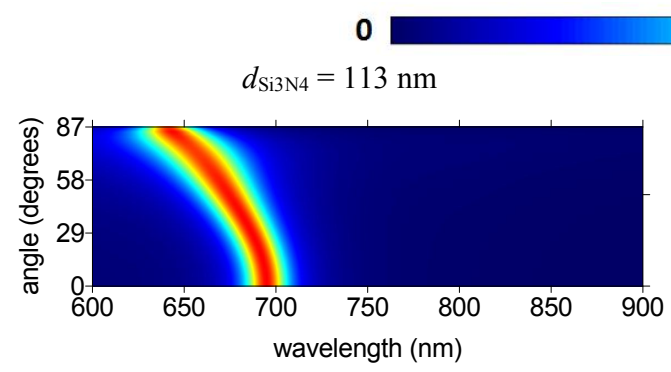

(a)

$d_{\mathrm{Si} 3 \mathrm{~N} 4}=133 \mathrm{~nm}$

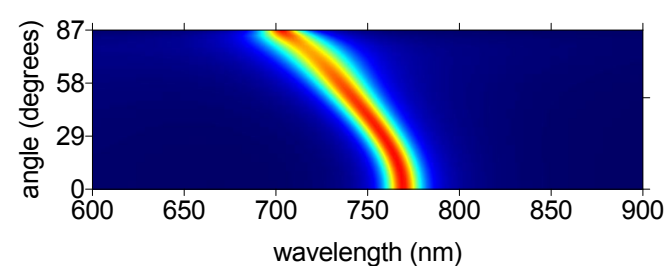

(c)

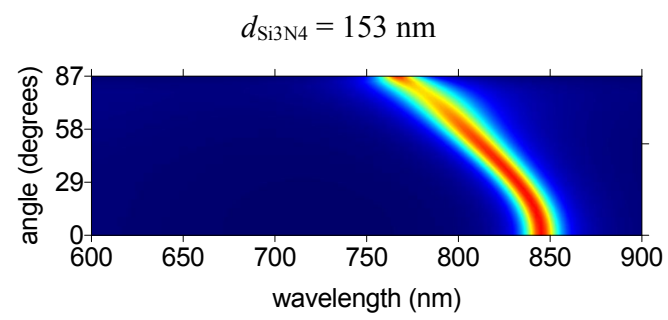

(e)

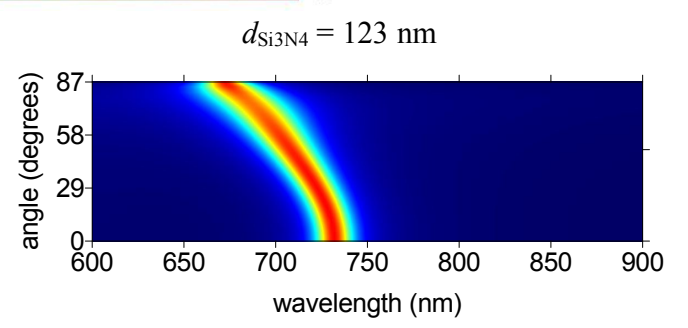

(b)

$d_{\mathrm{Si} 3 \mathrm{~N} 4}=143 \mathrm{~nm}$

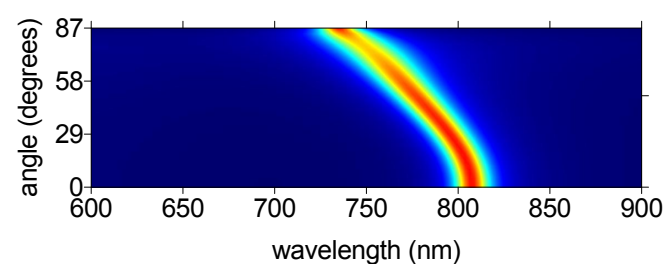

(d)

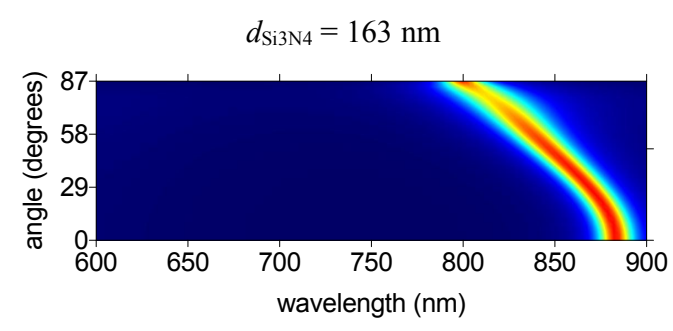

(f)

Fig. 8. Absorption as a function of the incidence angle and wavelength for TM polarization for several dielectric layer thicknesses.

It is also possible to observe that the absorption peak remain close to unity even if the oblique angle of the incidence is too large, for both polarizations. All these features and characteristics can be used in angular and polarization detection [6], [7], [21], [22].

\section{CONCLUSION}

In conclusion, we proposed and analyzed a resonant planar metal-dielectric-FP resonant absorber with narrow absorption width. Numerical results show near $100 \%$ absorption in the visible-infrared spectrum region. It has been shown that the resonance peaks of the structure can be related to the dielectric thickness using a linear function. The physical mechanism associated with the strong coupling was analyzed for TE and TM modes. We also studied the dependence of the absorption with the thickness of the top layer of Ag and a displacement towards the blue when the incident angle increases.

The fabrication process of this device is simple and can be performed using the electron beam evaporation method [12], [21] and [22]. The proposed structure can be used in many 
practical applications such as thermal emission, optical sensing, polarization detectors, among others.

\section{ACKNOWLEDGMENT}

The authors would like to thank CNPQ (Process: 309100/2018-6), CAPES, UFBA, and FAPESB (079/2016).

\section{REFERENCES}

[1] M. Aalizadeh, A. Khavasi, B. Butun, and E. Ozbay, "Large-Area, Cost-Effective, UltraBroadband Perfect Absorber Utilizing Manganese in MetalInsulator-Metal Structure," Sci. Rep., vol. 8, no. 9162, pp. 1-13, Jun. 2018.

[2] X. Dong and L. Chen, "Ultrabroadband Plasmonic Absorber Based on Biomimetic Compound Eye Structures," IEEE Photon. J., vol. 10, no. 1, pp. 1-7, Feb. 2018.

[3] Y. Ra'di, C. R. Simovski, and S. A. Tretyakov, "Thin perfect absorbers for electromagnetic waves: theory, design, and realizations," Phys. Rev. Appl., vol. 3, no.3, pp. 0370011-03700137, Mar. 2015.

[4] G. Fu, X. Liu, Z. Huang, J. Chen, and Z. Liu, "Metallic Metasurfaces for Light Absorbers," IEEE Photon. Technol. Lett., vol. 6, no. 1, pp. 47-50, Jan. 2017.

[5] N. Liu, M. Mesch, T. Weiss, M. Hentschel, and H. Giessen, "Infrared perfect absorber and its application as plasmonic sensor," Nano Lett., vol. 10, no. 7, pp. 2342-2348, Jun. 2010.

[6] B. H. Woo, I. Seo, E. Lee, S. An, H. Jeong, and Y. C. Jun, "Angle-dependent optical perfect absorption and enhanced photoluminescence in excitonic thin films," Opt. Exp., vol. 25, no. 23, pp. 28619-28629, Nov. 2017.

[7] D. Zhao, L. Meng, and H. Gong, "Ultra-narrow-band light dissipation by a stack of lamellar silver and alumina," Appl. Phys. Lett., vol. 104, no. 22, pp. 1-7, May. 2014.

[8] J. Zhou, Z. Liu, X. Liu, G. Fu, G. Liu, J. Chen, C. Wang, H. Zhang and M. Hong, "Metamaterial and nanomaterial electromagnetic wave absorbers: structures, properties and applications," J. Mater. Chem. C, vol. 8, no. 37, pp. 1276812794, Mar. 2020.

[9] Z. Liu, G. Liu, G. Fu, X. Liu, and Y. Wang, "Multi-band light perfect absorption by a metal layer-coupled dielectric metamaterial," Opt. Exp., vol. 24, no. 5, pp. 5020-5025, Feb. 2016.

[10] G. Liu, Y. Nie, G. Fu, X. Liu, Y. Liu, L. Tang and Z. Liu, "Semiconductor meta-surface based perfect light absorber," Nanotech., vol. 28, no. 16, 165202, Mar. 2017.

[11] B. Tang, Z. Li, E. Palacios, Z. Liu, S. Butun and K. Aydin, "Chiral-Selective Plasmonic Metasurface Absorbers Operating at Visible Frequencies," IEEE Photon. Technol. Lett., vol. 29, no. 3, pp. 295-298, Feb. 2017.

[12] X. Tu, C. Jiang, P. Xiao, L. Kang, S. Zhai, Z. Jiang, R. Su, X. Jia, L. Zhang, J. Chen, and P. Wu, "Investigation of antenna-coupled $\mathrm{Nb} 5 \mathrm{~N} 6$ microbolometer THz detector with substrate resonant cavity," Opt. Exp., vol. 26, no. 7, pp. 8990-8997, Apr. 2018.

[13] I. L. Gomes de Souza, V. F. Rodriguez-Esquerre, and D. F. Rêgo, "Wide-angle filters based on nanoresonators for the visible spectrum," Appl. Opt., vol. 57, no. 23, pp. 6755-6759, Jul. 2018.

[14] S. Shu, Z. Li, and Y. Y. Li, "Triple-layer Fabry-Perot absorber with nearperfect absorption in visible and near-infrared regime," Opt. Exp., vol. 21, no.21, pp. 25307-25315, Oct. 2013.

[15] Y. Zhan, K. Wu, C. Zhang, S. Wu, and X. Li, "Infrared hot-carrier photodetection based on planar perfect absorber," Opt Lett. vol. 40, no. 18, pp. 4261-4264, Sep. 2015.

[16] A. Soni, S. Purohit, and R. S. Hegde, "Multilayered Aluminum Plasmonic Metasurfaces for Ultraviolet Bandpass Filtering," IEEE Photon. Technol. Lett., vol. 29, no. 1, pp. 110-113, Jan. 2017.

[17] H. Shin, M. Yanik, and S. Fan, "Omnidirectional resonance in a metal-dielectric-metal geometry," Appl. Phys., vol. 84, no. 22, pp. 4421-4423, Apr. 2004.

[18] C. E. Rubio-Mercedes and H. E. Hernández-Figueroa, "Padé boundary conditions for the finite-element solution of arbitrary planar junctions,” J. Lightw. Technol., vol. 22, no. 2, pp. 669-676, Feb. 2004.

[19] K. Luke, Y. Okawachi, M. R. E. Lamont, A. L. Gaeta, and M. Lipson, "Broadband mid-infrared frequency comb generation in a Si3N4 microresonator," Opt. Lett., vol. 40, no. 21, pp. 4823-4826, Nov. 2015.

[20] P. B. Johnson and R. W. Christy, "Optical constants of the noble metals," Phys. Rev., vol. 6, no. 12, pp. 4370-4379, Dec. 1972.

[21] Z. Li, S. Butun, and K. Aydin, "Large-Area, Lithography-Free Super Absorbers and Color Filters at Visible Frequencies Using Ultrathin Metallic Films," ACS Phot., vol. 2, no. 2, pp. 183-188, Jan. 2015.

[22] K. Mao, W. Shen, C. Yang, X. Fang, W. Yuan, Y. Zhang, and X. Liu, "Angle insensitive color filters in transmission covering the visible region," Sci. Rep. vol. 6, no. 19289, pp. 1-7, Jan. 2016. 